\title{
CONSTRUCTING APPROXIMATE INVERSE SYSTEMS OF METRIC SPACES
}

By

\section{G. Charalambous}

\begin{abstract}
We formulate a theorem which provides a sufficient condition under which we can construct new approximate inverse systems from old. The result is at the heart of many constructions in the theory of approximate inverse systems and offers a unified approach to several important results in Topology such as Brown's approximation theorem, McCord's embedding theorem and results on expansion of $\Pi$-like spaces into inverse limits of spaces from $\Pi$.
\end{abstract}

\section{Definitions and Background}

The spaces considered in this paper, unless otherwise indicated, are metric and the maps between them are uniformly continuous. The symbol $d$ as a rule denotes a metric on the space indicated by the context. For functions $f, g$ : $X \rightarrow Y$ into a metric space, however, $d(f, g)$ denotes the supremum of the set $\{d(f(x), g(x)): x \in X\}$, which may take the value $\infty$. Moreover, for a non-empty subset $A$ of a metric space $X$ and a point $x$ of $X, d(x, A)$ denotes the infimum of the set $\{d(x, a): a \in A\}$.

An approximate inverse system, usually abbreviated to $A I S$, of metric spaces (and uniformly continuous maps) $\left(X_{\alpha}, p_{\alpha \beta}, A\right)$ consists of a directed set $A$ with respect to a transitive and anti-reflexive relation $<, a$ metric space $X_{\alpha}$ for each $\alpha$ in A and, for $\alpha<\beta$ in $A$, a uniformly continuous map $p_{\alpha \beta}: X_{\beta} \rightarrow X_{\alpha}$ satisfying the following condition.

(AIS) For each $\alpha$ in $A$ and each positive $\varepsilon$, there is $\alpha^{\prime}$ in $A$ such that $\alpha<\alpha^{\prime}$ and, for $\alpha^{\prime}<\beta<\gamma, d\left(p_{\alpha \gamma}, p_{\alpha \beta} p_{\beta \gamma}\right)<\varepsilon$.

The limit space $X$ of an $A I S\left(X_{\alpha}, p_{\alpha \beta}, A\right)$ of metric spaces is the subspace of the product $\prod\left\{X_{\alpha}: \alpha \in A\right\}$ consisting of all points $\left(x_{\alpha}\right)$ such that each $x_{\alpha}$ is the

Mathematics Subject Classification. 54B35, 54C35, 54E15, 54E35, 54F45.

Key words and phrases: inverse limit, approximate inverse limit; compact, metric and uniform spaces. Received March 24, 1998

Revised May 18, 1999 
limit of the net $\left\{p_{\alpha \beta}\left(x_{\beta}\right): \alpha<\beta\right\}$. Of course, if $A$ is uncountable, the limit space $X$ may not be metrizable. The map $p_{\alpha}: X \rightarrow X_{\alpha}$ that sends $\left(x_{\alpha}\right)$ to $x_{\alpha}$ will be referred to as the $\alpha^{\text {th }}$ canonical projection. It is important to bear in mind that the uniform covers of $X$ are those that can be refined by one of the form $p_{\alpha}^{-1}\left(\mathscr{U}_{\alpha}\right)$, where $\mathscr{U}_{\alpha}$ is a uniform cover of $X_{\alpha}$ and $\alpha$ ranges over any given cofinal subset of $A$ [3, proposition 4].

Approximate inverse systems of uniform spaces, satisfying the appropriate modification of $(A I S)$, were first considered in [3], following the introduction of approximate inverse systems of compacta by Mardešić and Rubin in [8]. Mardešić and Watanabe generalised approximate systems to arbitrary topological spaces [12]. The approximate systems studied in [8 and 12] were required to satisfy, apart from an appropriate modification of $(A I S)$, two extra conditions stipulating the existence of normal coverings (meshes) with certain properties. Such systems are now called gauged approximate systems and the term approximate inverse systems is used for systems satisfying only the appropriate modification of $(A I S)$ [7]. The much simpler notion of approximate inverse system is quite an adequate tool, however, in a variety of topological situations. In fact, with each $A I S$ of topological spaces one can associate an induced gauged $A I S$ consisting of the same spaces and maps and sharing the important properties of the original AIS [7, 16]. In particular, the two systems have the same limit space.

Let $\left(X_{\alpha}, p_{\alpha \beta}, A\right),\left(Y_{\alpha}, q_{\alpha \beta}, A\right)$ be approximate inverse systems of metric spaces with limit spaces $X, Y$ and canonical projections $p_{\alpha}, q_{\alpha}$. In this paper, a map $\left(h_{\alpha}\right):\left(X_{\alpha}, p_{\alpha \beta}, A\right) \rightarrow\left(Y_{\alpha}, q_{\alpha \beta}, A\right)$ will mean a collection of maps $h_{\alpha}: X_{\alpha} \rightarrow Y_{\alpha}$, $\alpha \in A$, such that for each $\alpha$ in $A$ and each positive $\varepsilon$, there is $\alpha^{\prime}$ in $A$ such that $\alpha<\alpha^{\prime}$ and, for $\alpha^{\prime}<\beta, d\left(h_{\alpha} p_{\alpha \beta}, q_{\alpha \beta} h_{\beta}\right)<\varepsilon$. In such a case, there is an induced map $h: X \rightarrow Y$ that sends $\left(x_{\alpha}\right)$ to $\left(h_{\alpha}\left(x_{\alpha}\right)\right)$. That $\left(h_{\alpha}\left(x_{\alpha}\right)\right)$ is a point of $Y$ follows from the continuity of $h_{\alpha}$ and the inequality

$$
d\left(h_{\alpha}\left(x_{\alpha}\right), q_{\alpha \beta} h_{\beta}\left(x_{\beta}\right)\right) \leq d\left(h_{\alpha}\left(x_{\alpha}\right), h_{\alpha} p_{\alpha \beta}\left(x_{\beta}\right)\right)+d\left(h_{\alpha} p_{\alpha \beta}\left(x_{\beta}\right), q_{\alpha \beta} h_{\beta}\left(\left(x_{\beta}\right)\right) .\right.
$$

That $h$ is uniformly continuous follows from the equality $q_{\alpha} h=h_{\alpha} p_{\alpha}$. Evidently, if each $h_{\alpha}$ is the identity on $X_{\alpha}$, then $h$ is the identity on $X$. Furthermore, if each $h_{\alpha}$ is an embedding, then so is $h$. For if $\mathscr{U}$ is a uniform cover of $X$, it is refined by $p_{\alpha}^{-1}\left(\mathscr{U}_{\alpha}\right)$ for some $\alpha$ in $A$ and some uniform cover $\mathscr{U}_{\alpha}$ of $X_{\alpha}$. As $h_{\alpha}$ is an embedding, $\mathscr{U}_{\alpha}$ is refined by $h_{\alpha}^{-1}\left(\mathscr{V}_{\alpha}\right)$ for some uniform cover $\mathscr{V}_{\alpha}$ of $Y_{\alpha}$. Hence $\mathscr{U}$ is refined by $h^{-1}\left(q_{\alpha}^{-1}\left(\mathscr{V}_{\alpha}\right)\right)=p_{\alpha}^{-1}\left(h_{\alpha}^{-1}\left(\mathscr{V}_{\alpha}\right)\right)$, and $h$ is an embedding.

Recall that an AIS $\left(X_{\alpha}, p_{\alpha \beta}, A\right)$ is called cofinite if $|\alpha|$, the number of predecessors of $\alpha$ in $A$, is finite for each element $\alpha$ of $A$. 


\section{A General Result}

THEOREM 1. Let $\left(X_{\alpha}, p_{\alpha \beta}, A\right)$ be a cofinite AIS of metric spaces and uniformly continuous maps. Let $H_{\alpha}, \alpha \in A$, be a non-empty collection of maps with domain $X_{\alpha}$ and $Q_{\alpha \beta}, \alpha<\beta$, a collection of maps with the following property.

$\left(^{*}\right)$ For a fixed $\beta$ in $A$ and $\varepsilon>0$, given for each $\alpha<\beta$, a map $h_{\alpha}: X_{\alpha} \rightarrow P_{\alpha}$ in $H_{\alpha}$, there exist a map $h_{\beta}: X_{\beta} \rightarrow P_{\beta}$ in $H_{\beta}$ and, for each $\alpha<\beta$, a map $q_{\alpha \beta}: P_{\beta} \rightarrow$ $P_{\alpha}$ in $Q_{\alpha \beta}$ such that $d\left(q_{\alpha \beta} h_{\beta}, h_{\alpha} p_{\alpha \beta}\right)<\varepsilon$.

Then there are, for each $\alpha$ in $A$, a positive real number $\varepsilon_{\alpha}$ and a map $h_{\alpha}: X_{\alpha} \rightarrow$ $P_{\alpha}$ in $H_{\alpha}$ and, for each $\beta>\alpha$, a map $q_{\alpha \beta}: P_{\beta} \rightarrow P_{\alpha}$ in $Q_{\alpha \beta}$ such that, if $Y_{\alpha}=$ $\left\{x \in P_{\alpha}: d\left(x, h_{\alpha}\left(X_{\alpha}\right)\right) \leq \varepsilon_{\alpha}\right\}$, then $q_{\alpha \beta}$ maps $Y_{\beta}$ into $Y_{\alpha},\left(Y_{\alpha}, q_{\alpha \beta}, A\right)$ is an AIS and $\left(h_{\alpha}\right)$ is a map from $\left(X_{\alpha}, p_{\alpha \beta}, A\right)$ to $\left(Y_{\alpha}, q_{\alpha \beta}, A\right)$. For $|\alpha|=0, h_{\alpha}$ and $\varepsilon_{\alpha}$ may be chosen arbitrarily.

Proof. By induction on $|\beta|$, we choose, for each $\beta$ in $A$, a positive real number $\varepsilon_{\beta}$ and maps $h_{\beta}: X_{\beta} \rightarrow P_{\beta}$ in $H_{\beta}$ and $q_{\alpha \beta}: P_{\beta} \rightarrow P_{\alpha}$ in $Q_{\alpha \beta}$ for each $\alpha<\beta$ such that

$$
\begin{gathered}
d\left(q_{\alpha \beta} h_{\beta}, h_{\alpha} p_{\alpha \beta}\right)<\min \left\{2^{-|\beta|}, \varepsilon_{\alpha} / 2\right\} \text { and } \\
d(x, y)<2 \varepsilon_{\beta} \text { in } P_{\beta} \Rightarrow d\left(q_{\alpha \beta}(x), q_{\alpha \beta}(y)\right)<\min \left\{2^{-|\beta|}, \varepsilon_{\alpha} / 2\right\} \text { in } P_{\alpha} .
\end{gathered}
$$

If $|\beta|=0$, we choose $h_{\beta}$ and $\varepsilon_{\beta}$ arbitrarily. Assuming we have chosen $\varepsilon_{\delta}, h_{\delta}$ and $q_{\gamma \delta}$ with the above properties, where $\alpha$ and $\beta$ have been replaced by $\gamma$ and $\delta$, respectively, for $|\gamma|$ and $|\delta|<|\beta|$, using $\left(^{*}\right)$ with $\varepsilon=\min \left\{2^{-|\beta|}, \varepsilon_{\alpha} / 2: \alpha<\beta\right\}$, we first choose maps $h_{\beta}: X_{\beta} \rightarrow P_{\beta}$ in $H_{\beta}$ and $q_{\alpha \beta}: P_{\beta} \rightarrow P_{\alpha}$ in $Q_{\alpha \beta}$ for each $\alpha<\beta$ that satisfy (1). Then, by the uniform continuity of $q_{\alpha \beta}$, we can choose $\varepsilon_{\beta}>0$ such that (2) is satisfied. This completes the construction of $\varepsilon_{\alpha}, h_{\alpha}$ and $q_{\alpha \beta}$.

Let $y$ be a point of $Y_{\beta}$. Then $d\left(y, h_{\beta}(x)\right)<2 \varepsilon_{\beta}$ for some point $x$ of $X_{\beta}$. By (2) and (1), respectively, we have

$$
\begin{gathered}
d\left(q_{\alpha \beta}(y), q_{\alpha \beta} h_{\beta}(x)\right)<\varepsilon_{\alpha} / 2 \text { and } \\
d\left(q_{\alpha \beta} h_{\beta}(x), h_{\alpha} p_{\alpha \beta}(x)\right)<\varepsilon_{\alpha} / 2 .
\end{gathered}
$$

Hence $d\left(q_{\alpha \beta}(y), h_{\alpha} p_{\alpha \beta}(x)\right)<\varepsilon_{\alpha}$, and $d\left(q_{\alpha \beta}(y), h_{\alpha}\left(X_{\alpha}\right)\right) \leq \varepsilon_{\alpha}$. This assures that $q_{\alpha \beta}$ maps $Y_{\beta}$ into $Y_{\alpha}$, as required.

We next verify $(A I S)$ for the system $\left(Y_{\alpha}, q_{\alpha \beta}, A\right)$. Given $\alpha$ in $A$ and $\varepsilon>0$, because $\left(X_{\alpha}, p_{\alpha \beta}, A\right)$ is an $A I S$ and $h_{\alpha}$ is uniformly continuous, there is $\alpha^{\prime}$ in $A$ such that $\alpha<\alpha^{\prime}, 2^{-\left|\alpha^{\prime}\right|}<\varepsilon / 5$ and for $\alpha^{\prime}<\beta<\gamma$, 


$$
d\left(h_{\alpha} p_{x \gamma}, h_{\alpha} p_{\alpha \beta} p_{\beta \gamma}\right)<\varepsilon / 2 .
$$

For $y$ in $Y_{\gamma}, x$ in $X_{\gamma}$ and $\alpha^{\prime}<\beta<\gamma$, we have by the triangle inequality,

$$
\begin{aligned}
d\left(q_{\alpha \gamma}(y)\right. & \left., q_{\alpha \beta} q_{\beta \gamma}(y)\right) \\
\leq & d\left(q_{\alpha \gamma}(y), q_{\alpha \gamma} h_{\gamma}(x)\right)+d\left(q_{\alpha \gamma} h_{\gamma}(x), h_{\alpha} p_{\alpha \gamma}(x)\right) \\
& +d\left(h_{\alpha} p_{\alpha \gamma}(x), h_{\alpha} p_{\alpha \beta} p_{\beta \gamma}(x)\right)+d\left(h_{\alpha} p_{\alpha \beta} p_{\beta \gamma}(x), q_{\alpha \beta} h_{\beta} p_{\beta \gamma}(x)\right) \\
& +d\left(q_{\alpha \beta} h_{\beta} p_{\beta \gamma}(x), q_{\alpha \beta} q_{\beta \gamma} h_{\gamma}(x)\right)+d\left(q_{\alpha \beta} q_{\beta \gamma} h_{\gamma}(x), q_{\alpha \beta} q_{\beta \gamma}(y)\right) .
\end{aligned}
$$

Let us examine the terms of the right hand side of (6). By (5), the third term is less than $\varepsilon / 2$. By $(1)$, the second and fourth terms are smaller than $2^{-|\beta|} \leq$ $2^{-\left|\alpha^{\prime}\right|-1}<\varepsilon / 10$. By (1), we also have that $d\left(h_{\beta} p_{\beta \gamma}(x), q_{\beta \gamma} h_{\gamma}(x)\right)<\varepsilon_{\beta}$ and hence, by (2), the fifth term is less than $\varepsilon / 10$. Choosing $x$ so that $d\left(y, h_{\gamma}(x)\right)<2 \varepsilon_{\gamma}$, we have, by (2), that the first term is also smaller than $\varepsilon / 10$. Also, applying (2) twice, we have $d\left(q_{\beta \gamma} h_{\gamma}(x), q_{\beta \gamma}(y)\right)<2 \varepsilon_{\beta}$, and hence the sixth term is also less than $\varepsilon / 10$. Thus, $d\left(q_{\alpha \gamma}(y), q_{\alpha \beta} q_{\beta \gamma}(y)\right)<\varepsilon$ for all $\alpha^{\prime}<\beta<\gamma$ and all $y$ in $Y_{\gamma}$, and hence $\left(Y_{\alpha}, q_{\alpha \beta}, A\right)$ is an $A I S$.

Finally, given $\alpha$ in $A$ and $\varepsilon>0$, choose $\alpha^{\prime}$ in $A$ such that $\alpha<\alpha^{\prime}$ and $2^{-\left|\alpha^{\prime}\right|}<$ $\varepsilon$. Then, by (1), for $\alpha^{\prime}<\beta, d\left(q_{\alpha \beta} h_{\beta}, h_{\alpha} p_{\alpha \beta}\right)<2^{-|\beta|}<\varepsilon$. Hence $\left(h_{\alpha}\right)$ is a map from $\left(X_{\alpha}, p_{\alpha \beta}, A\right)$ to $\left(Y_{\alpha}, q_{\alpha \beta}, A\right)$, and this concludes the proof of the theorem.

\section{Brown's Theorem for Approximate Systems of Complete Metric Spaces}

We next note a generalisation to countable approximate systems of [3, proposition 8], which is repeatedly appealed to in the sequel.

Proposition 1. Let $\left(X_{i}, p_{i j}, A\right)$ be a countable AIS of complete metric spaces with limit space $X$. Then there is a uniform isomorphism $\pi: X \rightarrow Y$ onto the limit space of an inverse system $\left(X_{i}, \pi_{i j}, M\right)$, where $M$ is a cofinal sequence of $A$ and $\pi_{i j}=p_{i j}$ whenever $j$ is an immediate successor of $i$ in $M$. Given $m$ in $A$ and $\varepsilon>0$, $M$ can be chosen so that $m$ is its first element and $d\left(p_{m}, q \pi\right)<\varepsilon$, where $p_{m}$ : $X \rightarrow X_{m}$ and $q: Y \rightarrow X_{m}$ are the canonical projections.

Proof. When $A=N$, the set of positive integers, the result is [3, proposition 8]. In the general case, consider a cofinal sequence $B$ of $A$ containing $m$. $A s$ each $X_{i}$ is complete, by [3, proposition 7], the map that sends the point $\left(x_{i}\right)_{i \in A}$ of the limit space of $\left(X_{i}, p_{i j}, A\right)$ to the point $\left(x_{i}\right)_{i \in B}$ of the limit space of the approximate inverse sequence $\left(X_{i}, p_{i j}, B\right)$ is an isomorphism. It suffices therefore to apply [3, proposition 8] to $\left(X_{i}, p_{i j}, B\right)$. 
Let $f: X \rightarrow Y$ be a function between metric spaces and $\mathscr{H}$ a collection of functions whose domain and range contain $X$ and $Y$, respectively. We say that $f$ can be approximated by elements of $\mathscr{H}$ if for each positive $\varepsilon$ there is some member $h$ in $\mathscr{H}$ such that $d(f(x), h(x))<\varepsilon$ for each $x$ in $X$. We say that $f$ is a near isomorphism if it can be approximated by uniform isomorphisms from $X$ to $Y$. As continuous functions on compact spaces are uniformly continuous, the notion of near isomorphism generalizes to metric spaces the term near homeomorphism of compact metric spaces. The composite of near isomorphisms is again a near isomorphism.

The following theorem generalizes to countable approximate inverse limits of complete metric spaces the result known as Brown's approximation theorem [2, theorem 4]. Several proofs exist in the literature for the case of inverse sequences of compact metric spaces [1, 11, 14].

THEOREM 2. Let $\left(X_{i}, f_{i j}, A\right)$ be a countable AIS of complete metric spaces and near isomorphisms with limit space $X$. Then $X$ is isomorphic to each $X_{i}$. In fact, each canonical projection $p_{i}: X \rightarrow X_{i}$ is a near isomorphism.

Proof. Let $B$ be a cofinal sequence of $A, Z$ the limit space of $\left(X_{i}, f_{i j}, B\right)$ and $g_{i}: Z \rightarrow X_{i}$ the canonical projection. As seen in the proof of proposition 1, we have an isomorphism $\sigma: X \rightarrow Z$ such that $p_{i}=g_{i} \sigma, i \in B$. As the first element of $B$ can be chosen arbitrarily, it can be seen that it suffices to prove the result when $A=N$. Then, in an obvious manner, theorem 1 supplies an $A I S\left(X_{i}, p_{i j}, N\right)$ of complete metric spaces and isomorphisms and a map $\left(h_{i}\right)$ from $\left(X_{i}, f_{i j}, N\right)$ to $\left(X_{i}, p_{i j}, N\right)$, where $h_{i}$ is the identity on $X_{i}$ for each $i$ in $N$. This special nature of $\left(h_{i}\right)$ assures that the limit space of $\left(X_{i}, p_{i j}, N\right)$ is $X$. Given $m$ in $A$ and $\varepsilon>0$, by proposition 1 , there is a subsequence $M=\left\{m, m_{2}, m_{3}, \ldots\right\}$ of $N$, an inverse system $\left(X_{i}, \pi_{i j}, M\right)$ with limit space $Y$ and a uniform isomorphism $\pi: X \rightarrow Y$, such that $\pi_{i j}=p_{i j}$ whenever $j$ is an immediate successor of $i$ in $M$ and $d\left(p_{m}, q \pi\right)<\varepsilon$, where $q: Y \rightarrow X_{m}$ is the canonical projection. Evidently, being the composite of isomorphisms, each $\pi_{i j}$ is an isomorphism. Hence each projection $Y \rightarrow X_{m_{i}}$ is an isomorphism. Therefore $X$ is isomorphic to $X_{m}$ and, in fact, $p_{m}$ : $X \rightarrow X_{m}$ is a near isomorphism.

The following example shows that in theorem 2 the assumption of completeness is not redundant. It also shows that, in sharp contrast to what happens with inverse sequences, an approximate inverse sequence of infinite metric spaces and isomorphisms may have empty limit. 
EXAmple 1. Let $Q=\left\{q_{1}, q_{2}, \ldots\right\}$ be the set of all rationals in the interior $(0,1)$ of the unit interval $I=[0,1]$. Recall that if $A, B$ are countable dense subsets of nontrivial open intervals $I_{1}, I_{2}$, respectively, then there is a strictly increasing $f: I_{1} \rightarrow I_{2}$ that takes $A$ onto $B$. Thus, given $q$ in $Q$, there is an isomorphism $f: I \rightarrow I$ that takes $Q$ onto $Q-\{q\}$. Then, given $\varepsilon>0$, by considering an open interval $J$ around $q$ of length $\varepsilon$, we can construct an isomorphism $g: I \rightarrow I$ such that $g$ takes $Q-\{q\}$ onto $Q, J$ onto $J$ and leaves every point outside $J$ fixed. Clearly, $d(f, g f)<\varepsilon$ and the restriction $f \mid Q: Q \rightarrow Q$ is a near isomorphism.

We can, therefore, construct by induction injective near isomorphisms $f_{i}: Q$ $\rightarrow Q, i \in N$, such that $f_{1}(Q)=Q-\left\{q_{1}\right\}$ and $f_{i}(Q)=f_{1 i}^{-1}\left(Q-\left\{q_{i}\right\}\right)$ for $i>1$, where, for $n<m, f_{n m}$ denotes the composite of $f_{n}, f_{n+1}, \ldots, f_{m-1}$. Consider now the inverse sequence $\left(X_{i}, f_{i j}, N\right)$, where $X_{i}=Q$. Its limit is readily seen to be empty. As each $f_{i j}$ is a near isomorphism, by theorem 1 , there is an approximate inverse sequence $\left(X_{i}, q_{i j}, N\right)$ such that each $q_{i j}$ is an isomorphism $Q \rightarrow Q$ and yet its limit space is empty.

\section{McCord's Embedding Theorem for Approximate Systems}

THEOREM 3. Let $\left(X_{i}, p_{i j}, A\right)$ be a countable AIS of metric spaces with limit space $X$. For each $i$ in $A$, let $E_{i}$ be a metric space containing $X_{i}$ and $n_{i}$ the collection of all complete neighbourhoods of $X_{i}$ in $E_{i}$. Suppose that each $p_{i j}$ can be approximated by elements of $Q_{i j}$, the family of all uniform embeddings of members of $n_{j}$ into members of $n_{i}$. Then, for each $i$ in $A$, the canonical projection $p_{i}: X \rightarrow X_{i}$ can be approximated by embeddings of $X$ into elements of $n_{i}$.

Proof. By [3, proposition 7], for any cofinal subset $B$ of $A$, the map that sends the point $\left(x_{i}\right)_{i \in A}$ of $X$ to the point $\left(x_{i}\right)_{i \in B}$ of the limit space of the approximate inverse sequence $\left(X_{i}, p_{i j}, B\right)$ is an embedding. As $A$ is countable, it contains a cofinal sequence $B$. Thus, we see that we may assume that $A=N$. Then, in theorem 1, letting $H_{i}$ consist of all restrictions of the inclusion $h_{i}$ : $X_{i} \rightarrow E_{i}$ to members of $n_{i}$, we see that $\left({ }^{*}\right)$ is satisfied. Thus, we obtain an $A I S$ $\left(Y_{i}, q_{i j}, N\right)$, where $Y_{i}$ is a member of $n_{i}$ and $q_{i j}$ belongs to $Q_{i j}$, and a map $\left(h_{i}\right):\left(X_{i}, p_{i j}, N\right) \rightarrow\left(Y_{i}, q_{i j}, N\right)$. Consequently, $X$ is a subspace of the limit $Y$ of $\left(Y_{i}, q_{i j}, N\right)$ and each canonical projection $q_{i}: Y \rightarrow Y_{i}$ extends the canonical projection $p_{i}: X \rightarrow X_{i}$. Because each $Y_{i}$ is complete, given $m$ in $A$ and $\varepsilon>0$, by proposition 1 , there is a subsequence $M=\left\{m, m_{2}, m_{3}, \ldots\right\}$ of $N$, an inverse system $\left(Y_{i}, \pi_{i j}, M\right)$ with limit space $Z$ and a uniform isomorphism $\pi: Y \rightarrow Z$, such that $\pi_{i j}=q_{i j}$ whenever $j$ is an immediate successor of $i$ in $M$ and $d\left(q_{m}, q \pi\right)<$ 
$\varepsilon$, where $q: Z \rightarrow Y_{m}$ is the canonical projection. Evidently, being the composite of embeddings, each $\pi_{i j}$ is an embedding. Hence $q$ is an embedding and $p_{m}$ : $X \rightarrow X_{m}$ can be approximated by embeddings of $X$ into $Y_{m}$.

Corollary 1. Let $\left(X_{i}, p_{i j}, A\right)$ be a countable AIS with limit space $X$. Suppose that each $X_{i}$ is a subspace of a metric space $F_{i}$ and each $p_{i j}$ can be approximated by injective continuous functions from compact neighbourhoods of $X_{j}$ into compact neighbourhoods of $X_{i}$. Then $X$ is embeddable in each $F_{i}$.

Proof. Apply theorem 3, letting $E_{i}$ be a compact neighbourhood of $X_{i}$ in $F_{i}$ and noting that a compact space is complete and an injective continuous function on a compact space is a uniform embedding.

Corollary 2. Let $\left(X_{i}, p_{i j}, A\right)$ be a countable AIS of compact subspaces of a locally compact metric space $E$ with limit space $X$. Suppose that each $p_{i j}$ can be approximated by injective continuous functions from neighbourhoods of $X_{j}$ into $E$. Then $X$ is embeddable in $E$.

Proof. Because $E$ is locally compact and $X_{i}$ is compact, each neighbourhood of $X_{i}$ contains both a compact neighbourhood of $X_{i}$ as well as an $\varepsilon$-neighbourhood of $X_{i}$ for some positive $\varepsilon$. This readily implies that each $p_{i j}$ can be approximated by injective continuous functions from compact neighbourhoods of $X_{j}$ into compact neighbourhoods of $X_{i}$. The result, therefore, follows from corollary 1 .

The special case of corollary 2 for inverse sequences is the important theorem of McCord [13, theorem 2].

\section{M-Like Spaces}

In the sequel, $\Pi$ denotes a class of metric spaces. We call a uniform space $X$ $\Pi$-like if every uniform cover of $X$ can be refined by one of the form $f^{-1}(\mathscr{U})$, where $\mathscr{U}$ is a uniform cover of an element $P$ of $\Pi$ and $f: X \rightarrow P$ is a dense map, i.e., $f(X)$ is dense in $P$. In the most interesting case when $X$ is compact, this definition agrees with the original definition of $\Pi$-like in [9], which requires that the map $f$ be onto. If the map $f$ in the above definition is not necessarily dense, we call $X$ weakly $\Pi$-like. We will call a space $X$ weakly $\Pi$-approximable if it satisfies the following condition. 
$\left({ }^{* *}\right)$ There is a family $\mathscr{F}$ consisting of dense maps from $X$ into members of $\Pi$ such that

(i) every uniform cover of $X$ can be refined by one of the form $g^{-1}(\mathscr{U})$, where $g: X \rightarrow P$ is a member of $\mathscr{F}$ and $\mathscr{U}$ is a uniform cover of $P$, and

(ii) given $\varepsilon>0$ and a finite number of maps $f_{i}: X \rightarrow P_{i}$ in $\mathscr{F}$, there exist a map $f: X \rightarrow P$ in $\mathscr{F}$ and maps $p_{i}: P \rightarrow P_{i}$ such that $d\left(f_{i}, p_{i} f\right)<\varepsilon$.

The term П-approximable space will mean a space $X$ that satisfies the version of $\left(^{* *}\right)$ where the maps $p_{i}$ are surjective.

If $X$ is the limit space of an $A I S\left(X_{\alpha}, p_{\alpha \beta}, A\right)$ and $\mathscr{F}$ consists of the canonical projections $p_{\alpha}: X \rightarrow X_{\alpha}$, then $\mathscr{F}$ satisfies $\left({ }^{* *}\right)$ except that its elements may not be dense [3, propositions 2 and 4]. The following result may be seen as a partial converse.

THEOREM 4. A Hausdorff weakly П-approximable space $X$ is embeddable as a dense subspace in the limit of a cofinite AIS $\left(Y_{\alpha}, q_{\alpha \beta}, A\right)$ consisting of members of $\Pi$, where the cardinality of $A$ may be taken to be equal to the uniform weight of $X$. If $X$ is complete, the embedding is an isomorphism. If $X$ is $\Pi$-approximable, the bonding maps may be taken to be surjective.

Proof. Let A denote the set of finite subsets of a base $B$ of uniform covers of $X$, and identify a singleton in $A$ with the corresponding member of $B$. In $A$, write $\alpha \leq \beta$ iff $\alpha$ is a subset of $\beta$. Then $\left(X_{\alpha}, p_{\alpha \beta}, A\right)$, where each $X_{\alpha}$ is $X$ and each $p_{\alpha \beta}$ is the identity on $X$, is a cofinite $A I S$. In theorem 1 , for $\alpha$ in $B$, we let $H_{\alpha}$ consist of a single member $h_{\alpha}: X \rightarrow P$ of $\mathscr{F}$ into a member $P$ of $\Pi$ that contains a uniform cover $\mathscr{U}$ such that $h_{\alpha}^{-1}(\mathscr{U})$ refines $\alpha$. Otherwise, we let $H_{\alpha}=\mathscr{F}$. Finally, we let $Q_{\alpha \beta}$ consist of all maps with domain and range members of $\Pi$. Condition $\left({ }^{* *}\right)$ assures that condition $\left({ }^{*}\right)$ is satisfied, and theorem 1 produces an $A I S$ $\left(Y_{\alpha}, q_{\alpha \beta}, A\right)$ consisting of spaces from $\Pi$ and maps from $Q_{\alpha \beta}$, and a map $\left(h_{\alpha}\right)$ from $\left(X_{\alpha}, p_{\alpha \beta}, A\right)$ to $\left(Y_{\alpha}, q_{\alpha \beta}, A\right)$, where each $h_{\alpha}$ is dense. We therefore have a map $h: X \rightarrow Y$ such that $q_{\alpha} h=h_{\alpha}$, where $Y$ is the limit of $\left(Y_{\alpha}, q_{\alpha \beta}, A\right)$ and $q_{\alpha}$ denotes the canonical projection from $Y$ onto $Y_{\alpha}$. Now the uniform covers of $Y$ of the form $q_{\alpha}^{-1}(\mathscr{U})$, where $\mathscr{U}$ is a uniform cover of $Y_{\alpha}$ and $\alpha$ ranges over any cofinal subset of $A$, constitute a base for the uniform covers of $Y$ [3, proposition 4]. Hence the choice of $h_{\alpha}$ for $\alpha$ in $B$ guarantees that $h$ is an embedding, and, because each $h_{\alpha}$ is dense, so is $h$. Hence, if $X$ is complete, then $h$ is an isomorphism. Of course, $B$ may be taken to be of minimal cardinality as a uniform base of $X$, in which case the cardinality of $A$ equals the uniform weight of $X$. If 
$X$ is $\Pi$-approximable, $Q_{\alpha \beta}$ may be taken to consist of all surjective maps with domain and range members of $\Pi$, in which case all bonding maps $q_{\alpha \beta}$ will be surjective.

THEOREM 5. Let $\Pi$ consist of complete metric spaces. Then a complete, weakly $\Pi$-approximable metric space $X$ is the limit of an inverse sequence consisting of members of $\Pi$. If $X$ is $\Pi$-approximable, the bonding maps may be taken to be surjective.

Proof. By theorem 4, $X$ is the limit of a countable $A I S\left(X_{i}, p_{i j}, A\right)$ consisting of members of $\Pi$. By proposition $1, X$ is the limit space of an inverse sequence $\left(X_{i}, \pi_{i j}, M\right)$, where $M$ is a cofinal subset of $A$ and $\pi_{i j}=p_{i j}$ whenever $j$ is an immediate successor of $i$ in $M$. If $X$ is $\Pi$-approximable, by theorem 4, the bonding maps $p_{i j}$ may be taken to be surjective. Then, the bonding maps $\pi_{i j}$ of the sequence, being composites of surjective maps, are surjective.

REMARK 1. If $\Pi$ is the class of all compact polyhedra of dimension $\leq n$, then any compact Hausdorff space $X$ with $\operatorname{dim} X \leq n$ is $\Pi$-approximable [6, lemma 2; 8, 9, example 2]. In this case, theorem 5 yields a version of Freudenthal's original result that a compact metric space $X$ with $\operatorname{dim} X \leq n$ can be expanded into an inverse sequence of members of $\Pi$ with surjective bonding maps [5]. Also, Theorem 4 shows that a compact Hausdorff space $X$ with $\operatorname{dim} X \leq n$ can be expanded into an $A I S$ consisting of members of $\Pi$ and surjective bonding maps $[8$, theorem 5].

If $\Pi$ is a class of compact polyhedra with no isolated points, every compact Hausdorff $\Pi$-like space is $\Pi$-approximable [10, lemma 2], and theorem 4 shows that every compact Hausdorff $\Pi$-like space can be expanded into an $A I S$ consisting of members of $\Pi$ and surjective bonding maps [10, theorem 3]. Theorem 5 provides an expansion theorem for compact metric $\Pi$-like spaces into inverse sequences of members of $\Pi$ and surjective bonding maps.

Propositions 11, 12 and corollaries 4, 5 of [3] constitute applications of theorems 4,5 to topologically complete spaces. Note the minor corrections of these results made in [4].

For any class $\Pi$ of compact polyhedra, any $\Pi$-like continuum is $\Pi$ approximable [9, lemma 4], and theorem 5 yields theorem 1 of [9] that a $\Pi$-like continuum can be expanded into an inverse sequence of members of $\Pi$ with surjective bonding maps. There is an analogous expansion into an inverse sequence result due to Pasynkov [15, proposition 2] if $\Pi$ is a weakly hereditary class 
of compact polyhedra. This means that for each $P$ in $\Pi$ and each $\varepsilon>0$, there is a triangulation $K$ of $P$ of mesh $\leq \varepsilon$ such that any subpolyhedron of $P$ with respect to $K$ belongs to $\Pi$. Pasynkov's theorem is also a consequence of theorem 5 , as can be seen from the following result.

PROPOSITION 2. For each weakly hereditary class of compact polyhedra $\Pi$, every weakly $\Pi$-like space $X$ is $\Pi$-approximable.

For the proof, we require some preliminaries. Let a polyhedron $P$ be the realization of some finite simplicial complex $K$. Let $a$ be $a$ point of $P$. Then the projection $p_{a}: P-\{a\} \rightarrow P$ is defined as follows: on a simplex $s$ of $K, p_{a}$ is the identity when $a \notin s$ and, when $a \in s, p_{a}$ is the projection from a into the boundary of $s$. Evidently, $p_{a}$ is continuous and, in fact, uniformly continuous on the complement of any neighbourhood of $a$. For continuous functions $f_{1}, f_{2}: X \rightarrow P$, write $f_{1} \leq f_{2}$ if $f_{1}(x)$ is contained in the carrier of $f_{2}(x)$ for each $x$ in $X$. Evidently, $\leq$ is transitive.

Lemma 1. Let $f: X \rightarrow P$ be a continuous (resp. uniformly continuous) function into the realization $P$ of some finite simplicial complex $K$. Then there is a continuous (resp. uniformly continuous) $g: X \rightarrow Q$ onto a subpolyhedron $Q$ of $P$ with respect to $K$ such that $g \leq f$ and every continuous (resp. uniformly continuous) $h: X \rightarrow Q$ with $h \leq g$ is surjective (resp. dense).

ProOF. For a map $q: X \rightarrow P$, let $n(q)$ denote the number of simplices contained in the smallest subpolyhedron of $P$ containing $q(X)$. Clearly, $p \leq q$ implies $n(p) \leq n(q)$. Let $m=\min \{n(q): q \leq f\}$. Evidently, for some $g: X \rightarrow P$ with $g \leq f, n(g)=m$. Let $Q$ be the smallest subpolyhedron of $P$ containing $g(X)$, and consider $h: X \rightarrow Q$ with $h \leq g$. Suppose $h$ is not onto $Q$ and pick $a$ in $Q-h(X)$. Then $p_{a} h \leq h \leq g \leq f$ and $n\left(p_{a} h\right)<n(g)=m$. For $a$ cannot be an isolated vertex of $Q, a$ belongs to a proper simplex $s$ of $Q$ and $p_{a}$ collapses $s-\{a\}$ onto a proper face of $s$. It follows that $h$ is onto $Q$.

The uniform case is similar. If in the above argument $h(X)$ is not dense in $Q$, we choose $a$ in the interior of $Q-h(X)$ so that $p_{a}$ is uniform on $h(X)$.

In the sequel, a special triangulation $K$ of an element $P$ of $\Pi$ will mean a triangulation such that every subpolyhedron of $P$ with respect to $K$ belongs to $\Pi$.

ProOf OF PROPOSITION 2. Let $X$ be weakly $\Pi$-like. Let $\mathscr{F}$ consist of all dense maps $f: X \rightarrow P$, such that $P$ is in $\Pi, P$ is the realisation of some special triangulation $K$ and every map $g: X \rightarrow P$ with $g \leq f$ is dense. 
Any uniform cover $\mathscr{W}$ of $X$ has a refinement of the form $f^{-1}(\mathscr{U})$, where $f: X \rightarrow P$ is a map into a member $P$ of $\Pi$ and $\mathscr{U}$ is a uniform cover of $P$. Let $K$ be a special triangulation of $P$ such that the cover $\mathscr{V}$ consisting of the stars of the vertices of $K$ refines $\mathscr{U}$. By lemma 1 , there is a subpolyhedron $Q$ of $P$ and a map $g: X \rightarrow Q$ in $\mathscr{F}$ with $g \leq f$. Then, for any vertex $v$ of $P, g^{-1}(\operatorname{st}(v))$ is contained in $f^{-1}(\operatorname{st}(v))$. Hence, $g^{-1}(\mathscr{V})$ refines $\mathscr{W}$, and condition $(\mathrm{i})$ of $\left({ }^{* *}\right)$ is satisfied.

Let $\varepsilon>0$ and consider a finite number of maps $f_{i}: X \rightarrow P_{i}$ in $\mathscr{F}$. Let $K_{i}$ be a special triangulation of $P_{i}$ of mesh $<\varepsilon$ and $\mathscr{V}_{i}$ the cover consisting of the starts of its vertices. Then, by the previous paragraph, there is a map $f: X \rightarrow P$ in $\mathscr{F}$ such that, if $\mathscr{V}$ consists of the stars of the vertices of $P$, then $f^{-1}(\mathscr{V})$ refines each $f_{i}^{-1}\left(\mathscr{V}_{i}\right)$. For each vertex $v$ of $P$, pick a vertex $\varphi_{i}(v)$ of $P_{i}$ such that $f^{-1}(\operatorname{st}(v))$ is contained in $f_{i}^{-1}\left(\operatorname{st}\left(\varphi_{i}(v)\right)\right)$. Define $p_{i}: P \rightarrow P_{i}$ to be the simplicial map that sends $v$ to $\varphi_{i}(v)$. Then $p_{i} f \leq f_{i}$. Hence $d\left(f_{i}, p_{i} f\right)<\varepsilon$ and, because $f_{i}$ is in $\mathscr{F}, p_{i} f$ is dense. Hence $p_{i}$ is dense and, because $P$ is compact, $p_{i}$ is surjective. Thus, $X$ is $\Pi$-approximable.

A consequence of proposition 2 is the following result, which generalizes the compactification theorem for covering dimension.

COROLlaRY 3. Let $\Pi$ be a weakly hereditary class of compact polyhedra, and $X$ a topological space such that the covers of $X$ of the form $f^{-1}(\mathscr{U})$, where $f: X \rightarrow P$ is a continuous function into a member $P$ of $\Pi$ and $\mathscr{U}$ is a uniform cover of $P$, form a uniformity on $X$. Then $X$ has a $\Pi$-like compactification of the same weight as $X$.

Proof. $X$ with the obvious uniformity is weakly $\Pi$-like and, by proposition 2, П-approximable. Theorem 4 does the rest.

\section{References}

[1] F. D. Ancel, An alternative proof of M. Brown's theorem on inverse sequences of near homeomorphisms, Proceedings, Geometric Topology and Shape Theory, Dubrovnik 1986, Lecture Notes Math. 1283, Springer, Berlin, 1987, 1-2.

[2] M. Brown, Some applications of an approximation theorem for inverse limits, Proc. Amer. Math. Soc. 11 (1960), 478-483.

[3] M. G. Charalambous, Approximate inverse systems of uniform spaces and an application of inverse systems, Comment. Math. Univ. Carolinae 32 (1991), 551-565.

[4] M. G. Charalambous, A note on the non-emptiness of the limit of approximate systems, Comment. Math. Univ. Carolinae 37 (1996), 155-157.

[ 5 ] H. Freudenthal, Entwicklungen von Räumen and ihren gruppen, Comp. Math. 4 (1937), 145234. 
[6] S. Mardešić, On covering dimension and inverse limits of compact spaces, Illinois J. Math. 4 (1960), 278-291.

[7] S. Mardešić, On approximate inverse systems and resolutions, Fund. Math. 142 (1993), $241-255$.

[ 8 ] S. Mardešić and L. R. Rubin, Approximate inverse systems of compacta and covering dimension, Pacific J. Math. 138 (1989), 129-144.

[9] S. Mardešić and J. Segal, e-mappings onto polyhedra, Trans. Amer. Math. Soc. 109 (1963), 146164.

[10] S. Mardešić and J. Segal, $\mathscr{P}$-like continua and approximate inverse limits, Math. Japonica 33 (1988), 895-908.

[11] S. Mardešić and J. Segal, Stability of almost commutative inverse systems of compacta, Topology and Appl. 31 (1989), 285-299.

[12] S. Mardešić and T. Watanabe, Approximate resolutions of spaces and mappings, Glas. Mat. 24 (1989), 583-633.

[13] M. C. McCord, Embedding $\mathscr{P}$-like compacta in manifolds, Canadian J. Math. 19 (1967), 321332.

[14] J. van Mill, Infinite-Dimensional Topology, Prerequisites and Introduction, North-Holland, Amsterdam, 1989.

[15] B. A. Pasynkov, On universal compacta, Russian Math. Surveys 21 (1966), 77-86.

[16] N. Uglešić, A simple construction of meshes in approximate systems, Tsukuba J. Math. 19 (1995), 219-232.

Department of Mathematics

University of the Aegean,

Karlovassi 83200 Samos

Greece

e-mail: mcha@aegean.gr 